\title{
BLOQUEOS CON ANESTESIA LOCAL EN CIRUGIAA MENOR
} LOCAL ANESTHESIA FOR MINOR SURGERY

Natalia Morales Brenes 1 Josué Serrano Calvo 2 Abdy Orozco Montoya 3

123 Médico General Trabajador independiente, Sanjosé Costa Rica

\section{RESUMEN}

Muchos de los casos que llegan al Primer nivel de Atención o Servicio de Emergencias por cirugías menores se pueden abordar de manera sencilla, utilizando técnicas correctas con la anestesia local, la cual es un procedimiento que no tiene mucha dificultad, es de corta duración, puede realizarse sobre tejidos superficiales o estructuras anatómicas que sean fáciles de acceder, donde generalmente no suelen ocurrir importantes complicaciones. Para ello utilizamos anestésicos locales, los cuales bloquean los impulsos que pueda transmitir cada fibra nerviosa y así poder reducir e inclusive eliminar la percepción del dolor.

Palabras clave: Anestesia local, bloqueos, cirugía menor, infiltración subcutánea.

\section{Cómo citar:}

Morales, N., Serrano Calvo, J., \& Orozco Montoya, A. (2021). Bloqueos con anestesia local en cirugía menor. Revista Ciencia Y Salud, 5(1), Pág. 37-46.

\section{ABSTRACT}

Many of the cases that reach the First Level of Care or Emergency Service due to minor surgeries can be addressed in a simple way, using correct techniques with local anaesthesia, which is a procedure that is not too difficult to perform, can be done in a short period of time and can perform on superficial tissues or anatomical structures that are easy to access, where important complications generally do not occur. For this we use local anaesthetics which block the impulses that each nerve fibre can transmit and thus be able to reduce and even eliminate the perception of pain.

Keywords: Local anesthesia, block, minor surgery, subcutaneous infiltation.

Recibido: 03/dic/2020

Aceptado: 23/ene/2021

Publicado: 15/feb/2021

\section{(c) (i) (9)(2)}




\section{CIENCIA\&SALUD}

\section{INTRODUCCIÓN}

Los anestésicos locales y regionales son herramientas esenciales para el tratamiento del dolor en el Primer nivel de Atención o Servicio de Emergencias. Los agentes pueden administrarse por vía tópica, por vía intravenosa, por infiltración directamente en el área a ser anestesiada, o en el área de los nervios periféricos para abastecer la región. Por lo tanto, un anestésico local en contacto con un tronco nervioso causa una parálisis reversible tanto sensorial como motora en el área inervada $(1,2)$.

\section{MÉTODOS}

Se realizó una búsqueda y escogencia de la literatura en las bases de datos referenciales: Access medicine, Scielo, UpToDate, Elsevier. Los criterios de inclusión fueron publicaciones con las palabras clave "bloqueo regional", "anestesia local", en el idioma de español e inglés.

\section{MECANISMO DE ACCIÓN DE LOS ANESTÉSICOS LOCALES}

Los anestésicos locales bloquean la conducción de manera reversible al disminuir o prevenir el gran aumento transitorio en la permeabilidad de las membranas excitables al $\mathrm{Na}+$ que normalmente se produce por una ligera despolarización de la membrana. Esta acción de los anestésicos locales se debe a su interacción directa con los canales de $\mathrm{Na}+$ dependientes de voltaje.

Los canales de $\mathrm{Na}+$ son proteínas de membrana integrales, ancladas en la membrana plasmática. Cuando los anestésicos locales se unen al canal de sodio, lo hacen impermeable al $\mathrm{Na}+$, estos factores reducen la probabilidad de propagación del potencial de acción y la conducción nerviosa finalmente falla $(2,3)$.

Secuencia en la acción de los anestésicos locales

1. Bloqueo simpático (vasodilatación y aumento de temperatura)

2. Pérdida de sensibilidad dolorosa y térmica

3. Pérdida propioceptiva

4. Pérdida de la sensación de tacto-presión

5. Parálisis motora (4)

\section{TIPOS DE ANESTÉSICOS LOCALES}

- Ésteres: sufren hidrólisis por acción de las colinesterasas plasmáticas.

- Amidas: sufren metabolismo por enzimas microsómicas hepáticas.

Los principales factores al elegir un anestésico local son (ver Tabla 1):

1. INICIO DE ACCIÓN: este va a depender de la pKa (pH al cual $50 \%$ del fármaco se encuentra ionizado y $50 \%$ no ionizado). Si el pH del entorno es mayor que la pKa del fármaco, un mayor porcentaje del fármaco se encontrará en la forma no ionizada, la cual difunde con mayor rapidez a través de las membranas lipídicas y con inicio de acción más breve. Así, los fármacos con pKa baja tienen un inicio de acción más rápido.

LA DURACIÓN: Los anestésicos locales altamente solubles en lípidos tienen una mayor duración de acción, porque se difunden más lentamente desde un ambiente rico en lípidos hasta el torrente sanguíneo acuoso. 


\begin{tabular}{|c|c|c|c|c|}
\hline Anestésico & Grupo & Inicio de acción & Duración & $\begin{array}{l}\text { Propiedades, efec- } \\
\text { tos secundarios } \\
\text { Procaína }\end{array}$ \\
\hline Procaína & Éster & Lento & Corta & $\begin{array}{l}\text { Alergénico, vasodi- } \\
\text { latación }\end{array}$ \\
\hline Tetracaína & Éster & Lento (>10min) & Larga (>60min) & $\begin{array}{l}\text { Elevada toxicidad } \\
\text { sistémica }\end{array}$ \\
\hline Lidocaína & Amida & Rápida (2-4 min) & $\begin{array}{l}\text { Intermedia } \\
60 \mathrm{~min})\end{array}$ & $\begin{array}{l}\text { Más habitual, vaso- } \\
\text { dilatación modera- } \\
\text { da }\end{array}$ \\
\hline Mepivacaína & Amida & Rápida (2-4 min) & $\begin{array}{l}\text { Intermedia } \\
60 \mathrm{~min})\end{array}$ & $\begin{array}{l}\text { Similar a la lidocaí- } \\
\text { na, vasodilatación } \\
\text { leve }\end{array}$ \\
\hline Prilocaína & Amida & Rápida (2-4 min) & $\begin{array}{l}\text { Intermedia } \\
60 \mathrm{~min})\end{array}$ & $\begin{array}{l}\text { Amida con menor } \\
\text { toxicidad sistémica, } \\
\text { metahemoglobine- } \\
\text { mia a dosis altas. }\end{array}$ \\
\hline Bupivacaína & Amida & $\begin{array}{l}\text { I n t e r m e d i a } \\
(>10 \text { min) }\end{array}$ & Larga (>60min) & $\begin{array}{l}\text { Separación del blo- } \\
\text { queo sensitivo del } \\
\text { motor. }\end{array}$ \\
\hline
\end{tabular}

2.POSIBLES EFECTOS TÓXICOS SISTÉMICOS: La toxicidad puede variar desde síntomas neurológicos sutiles a convulsiones resistentes al tratamiento y por último, a colapso cardiovascular. Un ejemplo es la inyección intravascular involuntaria de bupivacaína durante la anestesia regional, ya que esta puede llegar a producir toxicidad cardiovascular grave, incluyendo depresión del ventrículo izquierdo, bloqueo cardíaco auriculoventricular y arritmias potencialmente mortales como taquicardia ventricular y fibrilación, por lo que se considera más cardio tóxico que la lidocaína $(5,6)$.

Vía de administración de los anestésicos locales

ANESTESIA TÓPICA: Estos anestésicos se aplican sobre la piel intacta antes de la instrumentación de la dermis, en la mucosa intacta y sobre la piel abierta para el control del dolor o antes de la reparación de una herida (5).

Ejemplos de indicaciones de anestesia tópica en ciertas áreas de la medicina (4, 7):

- Oftalmología: cirugía ocular por cataratas o alteraciones de refracción, exploración ocular para valoración de úlceras corneales, extracción de cuerpos extraños

- $\quad$ Ginecología: biopsia de vulva, eliminación de condilomas

- Gastroenterología: Gastroscopía

- Urología: sondaje uretral, adherencias balanoprepuciales

- Pediatría: punción lumbar, suturas. Aunque en algunos casos, dependiendo del procedimiento y de la situación del paciente, se debe valorar la sedación o incluso el uso de anestesia general

- $\quad$ Cirugía menor: preanestesia de infiltración

ANESTESIA POR INFILTRACIÓN: se deposita el fármaco en el mismo tejido que se va a explorar y en el tejido suprayacente que debe incidirse para abordar la lesión. La infiltración para pequeñas incisiones y biopsias se hace inicialmente con un botón dérmico (habón), con agujas finas de calibres 25 a 30 (Figura 1.A). Seguidamente, se cambia la aguja por una de calibre 21 o 22 y por el mismo botón ya anes- 


\section{CIENCIA\&SALUD}

tesiado se infiltra el anestésico abarcando $3 \mathrm{~cm}$ alrededor del sitio donde se realizará dicho procedimiento. Cuando se trata de superficies extensas que requieren más de un botón dérmico, se realiza la infiltración en forma de rombo donde también se abarca cierta profundidad del tejido (Figura 1.B). La finalidad de este procedimiento es "bañar" las ramas nerviosas terminales para bloquear in situ la transmisión, en especial la de percepción del dolor (9).

Indicaciones de la anestesia por infiltración (8, 9):

- $\quad$ Lavado, desbridamiento o sutura de heridas profundas

- $\quad$ Extirpación de pequeños tumores de piel

- $\quad$ Quistes sebáceos, quistes sinoviales o lipomas

- $\quad$ Drenaje de abscesos

- $\quad$ Reducción e inmovilización de fracturas

- $\quad$ Realización de técnicas invasivas dolorosas: pericardiocentesis, toracocentesis, drenaje de neumotórax, paracentesis, punción lumbar y punción suprapúbica

\section{Figura $1(10)$}

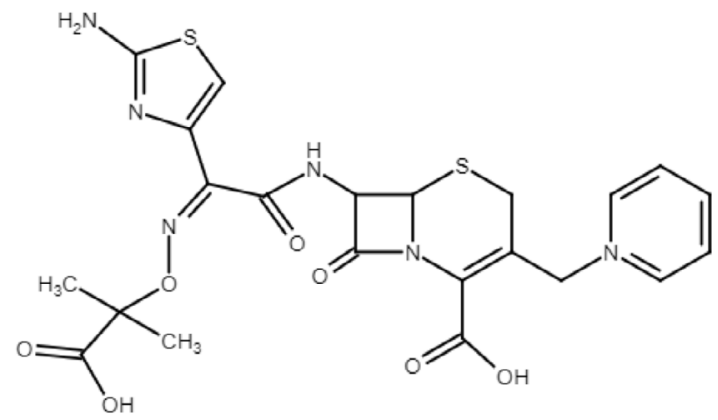

ANESTESIA REGIONAL: Las técnicas regionales incluyen el bloqueo neuroaxial, los anestésicos espinales y epidurales, los bloqueos nerviosos periféricos y los bloqueos del tronco, los cuales generalmente se realizan bajo guía ecográfica, para lograr una aplicación mucho más precisa del anestésico. Y por otro lado también se utiliza posoperatoriamente, ya que proporcionan un excelente control del dolor. Sin embargo, este estudio solo se enfoca en los bloqueos de nervios periféricos (15).

Dentro de las ventajas de esta técnica se encuentra una mayor duración de acción y ausencia de distorsión de la zona que se va a intervenir.

Sin embargo, pueden ocurrir inconvenientes ya que hay riesgo de daño neural directo (neuritis por punción neural) o por compresión a partir de hematomas.

Esto se puede minimizar si tenemos mucha práctica y cuidado a la hora de realizar la técnica y un buen conocimiento de la anatomía $(4,5)$.

Indicaciones de la anestesia regional:

- $\quad$ Sutura de heridas en dedos, barbilla y labio inferior

- Fracturas costales

- Onicocriptosis

- Luxaciones

- Abscesos

\section{ANESTÉSICOS LOCALES MÁS UTILIZADOS}

El anestésico local de uso más común en cirugía es el clorhidrato de lidocaína y viene en presentaciones de 0.5, 1, 2 y $5 \%$. Otra solución que se encuentra es con adrenalina al $2 \%$, la cual actúa como vasoconstrictor y se utiliza cuando se desea tener un efecto prolongado en regiones anatómicas que no tienen su circulación afectada. También se pueden obtener en forma de gel para aplicar en las mucosas o envasadas en rociadores para uso en aerosol. En ninguna de sus presentaciones se recomienda rebasar la dosis de $6 \mathrm{mg} / \mathrm{kg}$ (10) 
(Tabla 2).

\begin{tabular}{|l|l|l|}
\hline \multicolumn{2}{|l|}{ Tabla 2. Dosis máximas de algunos anestésicos locales (10) } \\
\hline Fármaco & Dosis & Para 70kg \\
\hline Lidocaína & $4 \mathrm{mg} / \mathrm{kg}$ & $280 \mathrm{mg}$ \\
\hline Bupivacaína & $2 \mathrm{mg} / \mathrm{kg}$ & $140 \mathrm{mg}$ \\
\hline Prilocaína & $5 \mathrm{mg} / \mathrm{kg}$ & $350 \mathrm{mg}$ \\
\hline Mepivacaína & $3.5 \mathrm{mg} / \mathrm{kg}$ & $245 \mathrm{mg}$ \\
\hline
\end{tabular}

\section{PUNTOS IMPORTANTES ANTES DE REALIZAR UN BLOQUEO $(4,5)$}

1. Se debe realizar una adecuada técnica aséptica.

2. Se debe valorar y documentar el estado neurovascular distal. La función vascular se verifica por el color, temperatura, llenado capilar y con la palpación de los pulsos. La función neurológica se verifi car por medio de la sensibilidad cutánea (dolor, tacto) y función motora (movimientos activos, fuerza).

3. Explicar al paciente la sensación de "pinchazo" y "picor", antes de realizar la infiltración.

4. Se debe aspirar para asegurar que no haya alguna estructura vascular comprometida, ya que esto aumenta la toxicidad del anestésico.

5. Se debe monitorizar los signos del paciente al igual que contar con recursos para el manejo de cuadros de hipersensibilidad o anafilaxia.

6. Prestar atención al momento de la punción ya que, si aparece un dolor intenso e irradiado, es por que se ha lesionado una terminal nerviosa y en este caso se tendría que retirar un poco la infiltración y continuar en otra zona.

\section{BLOQUEOS DIGITALES}

Estos bloqueos proporcionan anestesia en su totalidad, lo cual es beneficioso en heridas de dedos u ortejos, drenaje de paroniquia, extirpación o reparación de dedos o de la uña del primer ortejo y reducción por fracturas o luxaciones (5).

\section{MANO}

Los nervios digitales son terminaciones de los nervios mediano y cubital y estos se dividen en la palma distal en dos ramas terminales. Viajan junto con las arterias digitales justo a los lados de la vaina sinovial. Tienen ramas las cuales dan sensibilidad a la región dorsal y falange distal (11).

Figura 2 (5)<smiles>NC(=O)[C@@H]1CC[C@@H]2CN1C(=O)N2OS(=O)(=O)[O-]</smiles> 


\section{PROCEDIMIENTO:}

El paciente debe tener la mano y la muñeca en pronación, se realiza previa asepsia correspondiente y se introduce la aguja en la superficie dorsal de la porción proximal de la falange y se avanza hacia la superficie palmar, aspiramos para asegurar que no haya compromiso de alguna estructura vascular, se deposita 1cc de solución anestésica y se infiltra 1cc adicional mientras se retira la aguja en dirección posterior hacia la superficie de la piel (Figura 2. 1). Luego, introducimos la aguja en la misma ubicación, pero a través del dorso del dedo hacia lado opuesto y se inyecta 1cc de solución anestésica en el espacio subcutáneo (Figura 2.2). Se repite el proceso inicial de inyección en el lado opuesto del dedo (Figura 2.3). La aplicación del anestésico como ya se mencionó y se visualiza en la figura 2, bloquea ambos nervios digitales dorsal (a) y palmar (b). Al utilizar la secuencia mostrada, la primera infiltración proporciona alivio a las subsiguientes (5).

\section{PIE}

Uno de los bloqueos más utilizados es en los ortejos para realizar onicocriptosis también para toma de biopsias ungueales para completar el diagnóstico de alguna dermatosis, inclusive para extirpación o reparación de ortejos.

\section{PROCEDIMIENTO}

Cuando el paciente esté en la camilla, se realiza la asepsia adecuada, se infiltran 2cc del anestésico local a cada lado de la base de la falange proximal en la zona dorso medial y dorso lateral del dedo afectado (Figura 3), no debe contener epinefrina o algún otro vasoconstrictor que pudiese comprometer el buen aporte regional $(12,13)$.

Figura 3 (13)

A. Infiltración zona dorso medial

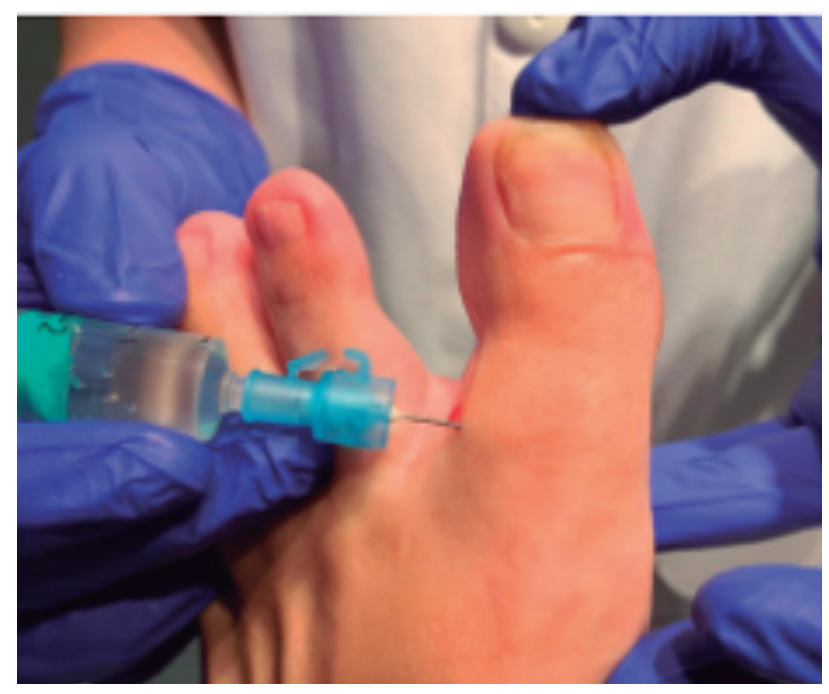


B. Infiltración zona dorso lateral

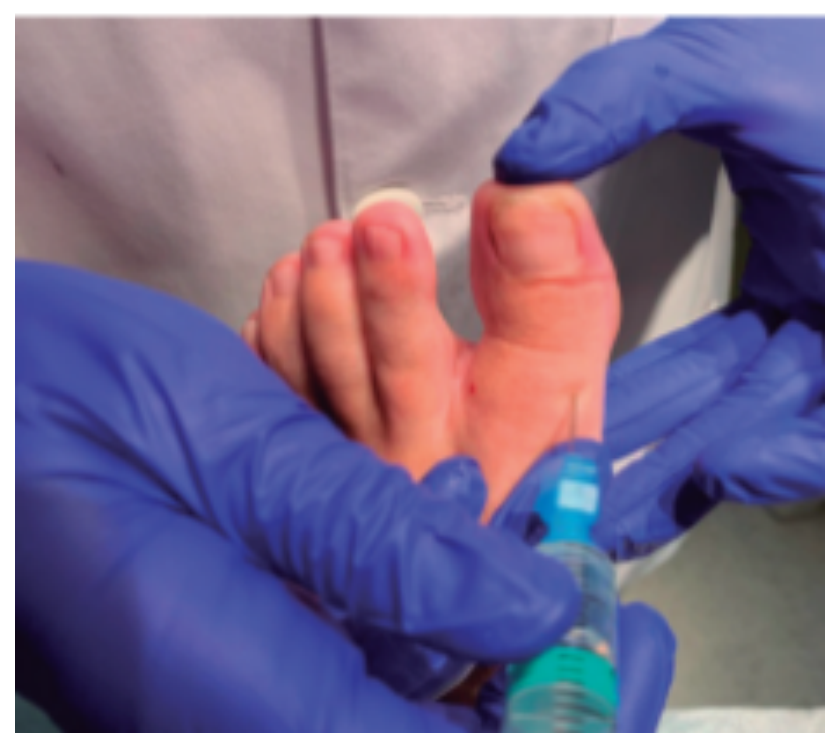

\section{BLOQUEO DE LOS NERVIOS INTERCOSTALES:}

Se realiza para procedimientos menores de la porción inferior del tórax o de la porción alta del abdomen. Se usa sobre todo en el alivio del dolor que producen las fracturas de las costillas.

Figura 4 (5)

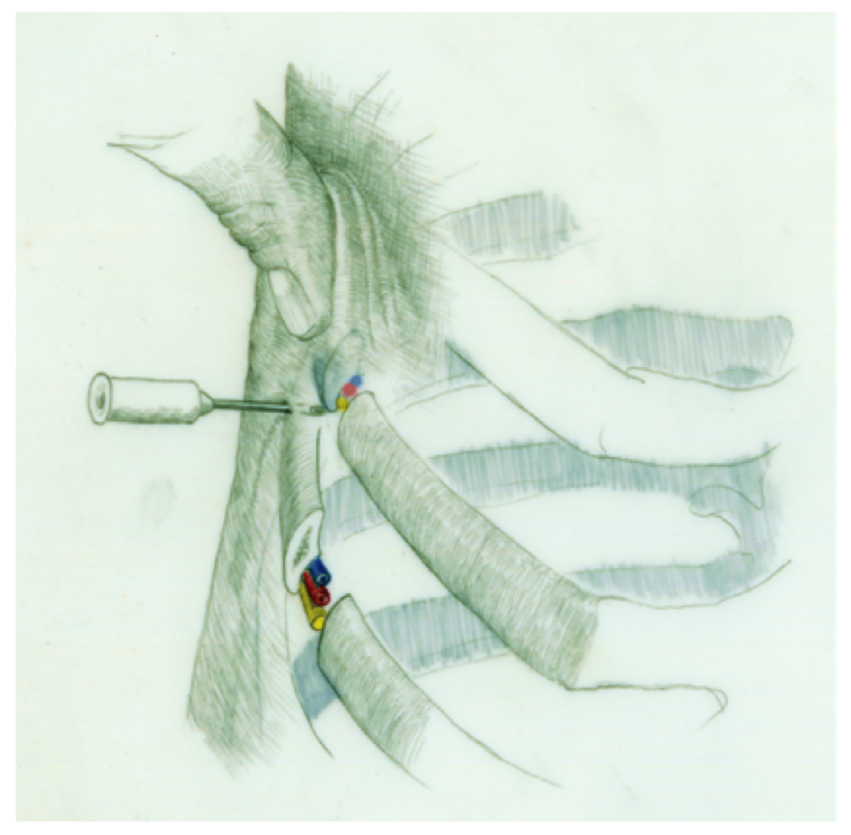

PROCEDIMIENTO:

Se infiltra en el punto intermedio entre la línea axilar posterior y las apófisis espinosas, a nivel del ángulo costal posterior (Figura 4). El bloqueo efectivo se consigue al infiltrar uno o dos espacios intercostales arriba y abajo del dermatoma implicado; el nervio intercostal se encuentra por debajo del borde inferior de la costilla, acompaña a la vena y a las arterias intercostales, que están arriba de él. 


\section{CIENCIA\&SALUD}

El paciente debe estar sentado, se realiza previa asepsia del área correspondiente, luego se hace un botón dérmico en el sitio seleccionado y se dirige la aguja número 22 a la porción inferior de la costilla.

En este sitio se aspira; si se obtiene aire, es que se ha penetrado la pleura; si es sangre, la aguja está en un vaso intercostal y se debe modificar la posición; sin aspiraciones obtiene más que vacío, se inyectan $5 c c$ de la solución anestésica (10).

\section{BLOQUEOS DE CARA}

El conocimiento de algunos bloqueos tronculares del área facial es de gran utilidad en la mayor parte de los procedimientos de cirugía menor. Dentro de las ventajas que proporciona el bloqueo regional está en que resulta menos dolorosa, el punto de bloqueo se encuentra alejado de las áreas a tratar, hecho importante en heridas contaminadas. También dentro de las ventajas se ahorra tiempo y permite trabajar sobre áreas extensas, con menos riesgo que la anestesia general (4).

Bloqueo del nervio supraorbitario

El nervio frontal es uno de los 3 pertenecientes a ramas terminales del nervio oftálmico (V1). Una de las dos ramas en las que se divide al llegar al borde orbitario superior es la supraorbitaria.

\section{PROCEDIMIENTO:}

El paciente se coloca en posición supina. El nervio supraorbitario sale a través del agujero supraorbitario (Figura 5), que se encuentra alineado con la pupila y por arriba del borde superior de la órbita.

El lugar donde se bloquea el nervio es inmediatamente superior a su salida, se crea una vesícula de anestesia en el tejido subcutáneo, justo por arriba de la ceja al nivel de la pupila. Una vez localizado el punto se introduce la aguja en un ángulo de $45^{\circ}$ con el bisel en sentido craneal, se depositan 2 a $3 \mathrm{ml}$ de solución anestésica en el espacio subcutáneo y luego se dirige la aguja en dirección interna a través del agujero depositando $1 \mathrm{ml}$ adicional en el nervio, se recomienda utilizar una aguja de 25 o 30 G; ya que es una zona sensible y susceptible de sangrado $(5,14)$.

\section{BLOQUEO DEL NERVIO INFRAORBITARIO}

El nervio infraorbitario es rama terminal del nervio maxilar (V2). Proporciona anestesia al párpado inferior, porción interna del carrillo, lado ipsolateral de la nariz y labio superior ipsolateral.

\section{PROCEDIMIENTO}

El paciente se coloca en decúbito supino. El nervio infraorbitario sale a través del agujero infraorbitario 5 a $10 \mathrm{~mm}$ por debajo de la porción media del borde orbitario y justo en dirección craneal (superior) con respecto al canino (diente 6 en el lado derecho y 11 en el lado izquierdo del paciente). La aguja (25-30 G) se orienta en un ángulo de $45^{\circ}$ medial y ligeramente hacia arriba (Figura 5); a continuación, se inyecta $1 \mathrm{ml}$ de solución anestésica $(5,14)$. 


\section{CIENCIA\&SALUD}

Figura 5 (5)

\section{Datos importantes que debemos recordar (4)}

- No sobrepasar las dosis máximas recomendadas

- $\quad$ Esperar el tiempo de latencia de 7-15 min.

- Usar vasoconstrictor, si no existe contraindicación

- $\quad$ Preguntar por alergias

- Aspirar la jeringa siempre antes de presionar el émbolo

- Disponer siempre de material y medicación de reanimación cardiopulmonar

- En los bloqueos regionales es imprescindible el conocimiento de la anatomía local

- $\quad$ No aplicar anestesia local en los lactantes menores de 3 meses de edad

\section{CONCLUSIONES}

Los bloqueos loco-regionales son técnicas que se pueden aprovechar tanto en la Atención de Primer Nivel como en el Servicio de Emergencias. Se mencionan los bloqueos más comunes en estas áreas, los cuales se pueden utilizar tanto para traumas, escisión de biopsias o tumores benignos, entre otros. Se requiere conocimiento de la anatomía local y una práctica constante para poder aumentar la agilidad.

\section{REFERENCIAS BIBLIOGRÁFICAS}

1. Mitchell M.S. (2018). Capítulo 7: Manejo del dolor agudo y procesos de sedación [Libro electrónico]. Cydulka R.K., \& Fitch M.T., \& Joing S.A., \& Wang V.J., \& Cline D.M., \& Ma O(Eds.), Manual de Urgencias Médicas de Tintinalli, 8e. McGraw-Hill. Disponible en: https://accessmedicina-mhmedical-com.binasss.idm.oclc.org/ content.aspx?bookid=2441\&sectionid=199578241.

2. Catterall W.A \& Mackie K (2019). Capítulo 22: Anestésicos locales [Libro electrónico]. Brunton L.L., \& Chabner B.A., \& Knollmann B.C.(Eds.), Goodman \& Gilman: Las Bases Farmacológicas De La Terapéutic, 13e. McGraw-Hill. Disponible en : https://accessmedicina-mhmedical-com.binasss.idm.oclc.org/content.aspx?bookid=2457\&sectionid $=202811174$.

3. Butterworth, J. F. (1990). Molecular mechanisms of local anesthesia: a review. PubMed. Disponible en: https://pubmed.ncbi.nlm.nih.gov/2157353/. 


\section{CIENCIA\&SALUD}

4. J.M. Arribas Blancoa, N. Rodríguez Pataa, B. Esteve Arrolaby M. Beltrán Martínc. (2001, octubre). Anestesia local y locorregional en cirugía menor. Elsevier, 27. Disponible en: https://www.elsevier.es/es-revista-medicina-familia-semergen-40-pdf-13020294.

5. Dillon D.C., \& Gibbs M.A. (2018). Capítulo 36: Anestesia local y regional [Libro electrónico]. Tintinalli J.E., \& Stapczynski J, \& Ma O, \& Yealy D.M., \& Meckler G.D., \& Cline D.M.(Eds.), Tintinalli. Medicina de urgencias, 8e. McGraw-Hill. Disponible en: https://accessmedicina-mhmedical-com.binasss.idm.oclc.org/content.aspx?bookid=2329\&sectionid=187779485.

6. Chapter 16: Local anesthetics. Butterworth IV J.F., \& Mackey D.C., \& Wasnick J.D.(Eds.), (2018). Morgan \& Mikhail's Clinical Anesthesiology, 6e. McGraw-Hill. Disponible en: https://accessmedicine-mhmedical-com. ezproxy.sibdi.ucr.ac.cr/content.aspx?bookid=2444\&sectionid=193558685.

7. Blanco, J.M. \& Pata, N. \& Castelló, Jose \& Marrodán, B. (2003). Uso de anestésicos tópico. FMC - Formación Médica Continuada en Atención Primaria. DOI: 10. 189-199. 10.1016/S1134-2072(03)75858-7.

8. P. García Bermejo, M. L. Tarrasó Gómez y J. L. Ruiz López. (2019). Módulo 2: Tratamiento del dolor, Tema 6: Técnicas analgésicas I [Libro electrónico]. Editorial Médica Panamericana. Disponible en: http://aula. campuspanamericana.com/_Cursos/Curso01254/Temario/M2T6/M2T6-Texto.pdf.

9. Martínez Dubois S (2012). Capítulo 8: Anestesia quirúrgica. Martínez Dubois S(Ed.), Cirugía bases del conocimiento quirúrgico y apoyo en trauma, 5e. McGraw-Hill. https://accessmedicina-mhmedical-com.binasss.idm.oclc.org/content. aspx?bookid=1466\&sectionid=101739584.

10. Capítulo 14: Anestesia [Libro electrónico]. García A. Archundia (Ed.), (2017). Cirugía 1. Educación quirúrgica, 6e. McGraw-Hill. Disponible en: https://accessmedicina-mhmedical-com.binasss.idm.oclc.org/content.aspx?bookid=2194\&sectionid=167842746.

11. Dr. Jorge Raúl Carrillo-Córdova, Dra. Sandra Ruiz-Beltrán, Dra. Hazel Bracho-Olvera, Dr. Yusef Jiménez-Murat, Acad. Dr. Raúl Carrillo-Esper, Cap. Nav. Juan Alberto Díaz Ponce-Medrano, Dr. Luis Daniel Carrillo-Córdova. (2017). Anestesia regional de miembro superior en cirugía plástica reconstructiva. Revista mexicana de anestesiología, 40, 38-46. Disponible en: https://www.medigraphic.com/pdfs/rma/cma-2017/ cma171f.pd

12. Arias L (2015). Patología ungueal. Saúl A(Ed.), Saúl. Lecciones de dermatología, 16e. McGraw-Hill. Disponible en: https://accessmedicina-mhmedical-com.binasss.idm.oclc.org/content.aspx?bookid=1537\&sectionid $=99046542$

13. Sergi Sánchez Hernández,A; Enrique Giralt de Veciana. (2020). Bloqueo digital anestésico con técnica v versus técnica $\mathrm{h}$ en onicocriptosis infectadas del primer dedo del pie. Revista ibero-americana de podología, 190-195. Disponible en: https://www.researchgate.net/publication/343972806_Bloqueo_digital_anestesico_con_tecnica_V_versus_tecnica_H_en_onicocriptosis_infectadas_del_primer_dedo_del_pie_Eficacia_Tecnica_en_V

14. S.Santos LasaosaaM.L.Cuadrado PérezbA.L.Guerrero PeralcM.Huerta VillanuevadJ.Porta-EtessambP.Pozo-RosicheJ.A.Parejaf. (2017). Guía consenso sobre técnicas de infiltración anestésica de nervios pericraneales. Sociedad Española de Neurocirugía, 32, 316-330. https://www.sciencedirect.com/science/article/ pii/SO21348531630076

15. Capítulo 46: Anestesia para pacientes quirúrgicos [Libro electrónico]. Nizamuddin J, \& O’Connor M (2020). Anestesia para pacientes quirúrgicos. Brunicardi F, \& Andersen D.K., \& Billiar T.R., \& Dunn D.L., \& Kao L.S., \& Hunter J.G., \& Matthews J.B., \& Pollock R.E.(Eds.), Schwartz. Principios de Cirugía, 11e. McGraw-Hill. Disponible en: https://accessmedicina-mhmedical-com.binasss.idm.oclc.org/content.aspx?bookid $=2958 \&$ sectionid $=252373240$ 